\title{
The Munich Compact Light Source: Flux Doubling and Source Position Stabilization At a Compact Inverse-Compton Synchrotron X-ray Source.
}

$\underline{\text { Benedikt Günther }}^{1,2,3^{*}}$, Martin Dierolf ${ }^{1,2}$, Martin Gifford ${ }^{4}$, Elena Eggl ${ }^{1,2}$, Bernhard Gleich ${ }^{2}$, Klaus Achterhold $^{1,2}$, Rod Loewen ${ }^{4}$ and Franz Pfeiffer ${ }^{1,2,5}$

1. Chair of Biomedical Physics, Department of Physics, Technical University of Munich, Garching, Germany.

2. Munich School of BioEngineering, Technical University of Munich, Garching, Germany.

3. Max-Planck-Institute of Quantum Optics, Garching, Germany

4. Lyncean Technologies Inc., Fremont, United States of America

5. Department of Diagnostic and Interventional Radiology, Technical University of Munich, Munich, Germany

* Corresponding author, benedikt.guenther@tum.de

Brilliant compact X-ray/XUV sources are mainly based on inverse-Compton scattering (ICS) or highharmonic generation (HHG) and enable the transfer of techniques developed at synchrotrons into a laboratory environment. Highly coherent XUV from HHG sources is used for, e.g., coherent diffractive imaging [1] and time-resolved photoelectron spectroscopy [2], while ICS sources are employed for, e.g., phase-contrast [3, 4], dynamic imaging [5] and micro-beam radiation therapy [6].

The Munich Compact Light Source (MuCLS) [7] belongs to the latter group and consists of an electron storage ring and a passive bow-tie laser resonator. The circulation direction in the laser resonator is opposite to the one in the electron storage ring generating a head-on collision of relativistic electrons with laser photons. This process of inverse-Compton scattering creates quasi-monochromatic X-ray radiation emitted into a small cone angle of $\sim 4 \mathrm{mrad}$ with a cut-off energy at $E_{\text {cut-off }}=4 \gamma E_{\text {laser }}$. Here, $\gamma$ is the Lorentz factor of the electrons, and $E_{\text {laser }}$ the energy of a laser photon. At the MuCLS, the electron energy is varied in order to adjust the X-ray energy between $15 \mathrm{keV}$ and $35 \mathrm{keV}$. Depending on the Xray energy, an X-ray flux up to $1.5 \cdot 10^{10} \mathrm{ph} / \mathrm{s}$ was available on a daily basis after commissioning of the source in 2015. Corresponding source characterization is given in [8].

With this source we could demonstrate advantages in biomedical imaging arising from the brilliance of the MuCLS $[3,4,6]$. Nevertheless, these studies also motivated us to pursue a further increase of X-ray flux, especially for time-sequence studies and micro-beam radiation therapy.

Accordingly, we installed a new laser system which more than doubled the available laser power from $14 \mathrm{~W}$ to $30 \mathrm{~W}$ and thus increases the power stored in the laser resonator from $\sim 120 \mathrm{~kW}$ to $\sim 300 \mathrm{~kW}$. This value is directly proportional to the number of laser photons, i.e. number of scatterers. As a result, the X-ray flux at $35 \mathrm{keV}$ is promoted above $3.0 \cdot 10^{10} \mathrm{ph} / \mathrm{s}$, more than doubling the initial value. Figure 1 displays the corresponding X-ray source parameters over a period of $10 \mathrm{~min}$. They were determined in the same way as in [8]. After initial optimization of the overlap of the laser and electron beam, the X-ray source position remains stable over short periods, but starts drifting on longer time scales typical for experiments, as depicted in Figure 2. This is due to relative shifts of electron and laser beam and causes a decline of the X-ray flux. The reason for the occurring shifts is attributed to temporal thermal gradients slightly affecting the laser resonator optics and shifting the orbit of the optical beam. In Figure 2, we 
exemplarily display a long run at $25 \mathrm{keV}$ to demonstrate the general necessity of a closed loop stabilization scheme for such X-ray sources.

We present the results of our characterization of the new laser system and the resulting X-ray parameters. In particular we discuss a set of feedback systems that are capable of significantly reducing the drift of the source position by more than one order of magnitude translating into a stable delivery of the increased flux [9].

With this performance upgrade enhanced in-vivo time-sequence X-ray imaging is possible in a laboratory environment [5]. Moreover, the increased X-ray flux renders flux-demanding techniques, like sub-micron X-ray microscopy or energy-dispersive X-ray absorption spectroscopy, feasible at ICS sources, such as the MuCLS [11].

References:

[1] R Sandberg et al, Phys. Rev. Lett. 99 (2007), 098103.

[2] L Cavalieri et al, Nature 449 (2007), p. 1029

[3] E Eggl et al, PNAS 112 (18) (2015), p. 5567.

[4] R Gradl et al, Scientific Reports 7 (2017), 4908.

[5] R Gradl et al, under Review.

[6] Burger et al, PLoS ONE 12 (10) (2017), e0186005.

[7] The Compact Light Source itself was developed by Lyncean Technologies Inc. (Fremont, USA) http://lynceantech.com

[8] E Eggl et al, Journal of Synchrotron Radiation 23 (2016), p. 1137.

[9] B. Günther et al, to be submitted

[10] E Eggl, PhD, Technical University of Munich (2017)

[11] We acknowledge financial support through the Center of Advanced Laser Applications (CALA) and the Cluster of Excellence of the German Research Foundation (DFG) Munich-Centre for Advanced Photonics (MAP).

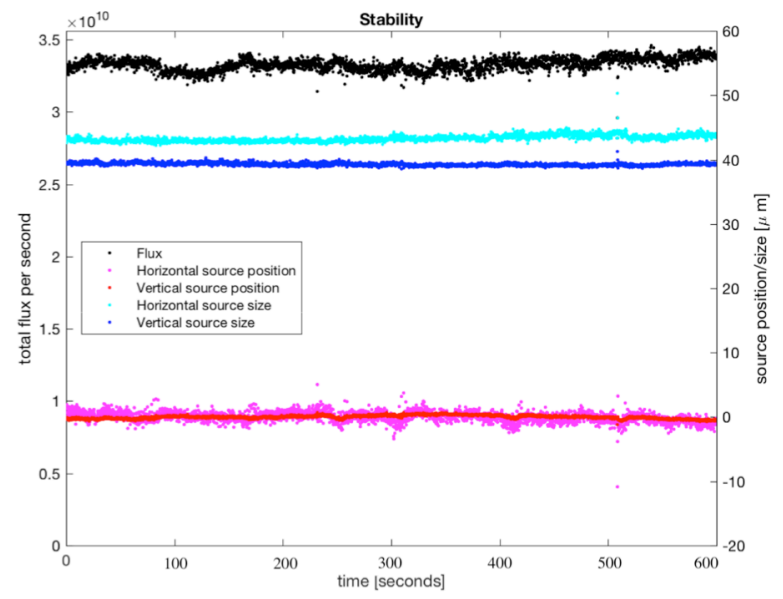

Figure. 1. X-ray source parameters of the MuCLS after the laser upgrade. At $35 \mathrm{keV}$ an X-ray flux above $3 \cdot 10^{10} \mathrm{ph} / \mathrm{s}$ is reached.

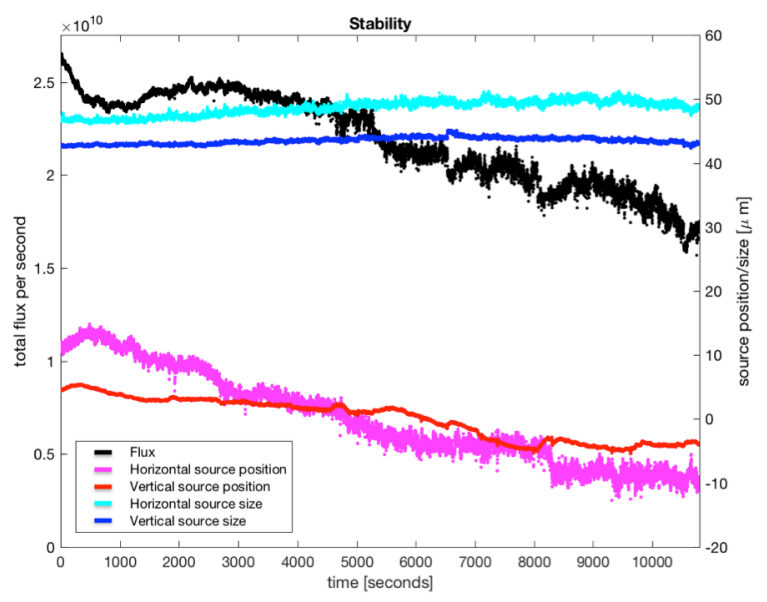

Figure. 2. X-ray source parameters over a period of 3 hours at $25 \mathrm{keV}$ exhibiting a decline on long time scales [10]. 